\title{
Dry gangrene of lower limbs in a child: an adverse drug reaction to chloramphenicol
}

\author{
Tanvir Samra $^{1 *}$, Lalita Chaudhary ${ }^{2}$, Ranvinder Kaur ${ }^{3}$ \\ ${ }^{I}$ Post graduate institute of medical education and research (PGIMER), Chandigarh, India \\ ${ }^{2,3}$ Lady Hardinge Medical College, New Delhi, India \\ *Corresponding author E-mail:drtanvirsamra@yahoo.co.in
}

\begin{abstract}
Drug induced vasculitis is a potentially life and limb threatening complication which can be precipitated by intake of virtually any pharmaceutical agent. It is a diagnosis of exclusion as definitive diagnostic and laboratory criteria have not been defined. Withdrawal of the offending agent is the definitive therapy but use of antithrombotic, vasodilatory and anti-inflammatory agents has been proven to be beneficial. We report a case of dry gangrene of bilateral lower limbs and digits of the upper limb due to DIV secondary to the intake of chloramphenicol in a child diagnosed with typhoid fever.
\end{abstract}

Keywords: Adverse Drug Reaction; Dry Gangrene; Chloramphenicol; Typhoid Fever; Drug Induced Vasculitis.

\section{Introduction}

An adverse drug reaction is defined as an appreciably harmful or unpleasant reaction, resulting from an intervention related to the use of a medicinal product, which predicts hazard from future administration and warrants prevention or specific treatment, or alteration of the dosage regimen, or withdrawal of the product (Edwards et al 2000). Drug induced vasculitis is a severe cutaneous drug reaction and this is the first case report to document its occurrence secondary to oral intake of chloramphenicol in a child diagnosed with typhoid fever.

\section{Case report}

A 10 year old male child weighing $15 \mathrm{~kg}$ presented to the paediatric surgical outpatient department with dry gangrene of bilateral lower limbs (Figure 1). Discoloration was first noticed in the digits of the lower limbs three days after commencement of chloramphenicol $(25 \mathrm{mg} / \mathrm{kg} /$ day in four divided doses) advised for treatment of typhoid fever by a private practitioner. Presentation to the hospital was delayed as the child lived in a remote village and thus the antibiotic had been consumed for a total duration of eight days i.e. 5 days after start of discoloration of toes and now it also involved the digits of upper limbs. A presumptive diagnosis of medium vessel vasculitis was made and the antibiotic was discontinued. The patient was afebrile and there was no documentation supporting a diagnosis of typhoid fever and thus no therapy was initiated for the same. He had no prior history of any systemic illness and his birth history and perinatal history were also normal. Routine laboratory investigations were done which were all negative except for mild anemia which was probably nutritional. He tested negative for anti-nutrophilic cytoplasmic antibody (ANCA) and had normal levels of $\mathrm{C}$ reactive protein (CRP). Anterior tibial, posterior tibial and dorsalis pedis arteries were not palpable on both the sides. Doppler confirmed absence of flow.

$\mathrm{He}$ was started on low molecular weight heparin (enoxaparin $1 \mathrm{mg} / \mathrm{kg}$ twice daily) but there was no improvement and he was scheduled for bilateral below knee amputation on the $12^{\text {th }}$ day.

\section{Discussion}

Vasculitis followed by dry gangrene secondary to use of chloramphenicol was the most probable etiology in our patient. "Vasculitis" is a term used for a group of disorders characterised by inflammation of the blood vessels (Jennette et al 1994). It can be further classified by the underlying etiology, location, type and size of the affected blood vessel. Apart from infectious etiologies (e.g. syphilitic aortitis) in which a clear cut diagnosis can be made majority of vasculitis are immune mediated and the trigger is often difficult to identify.

Drug induced vasculitis (DIV) accounts for 3\% of vasculitis (Doyle et al 2003). Distinction of drug induced and idiopathic small and medium vessel vasculitis may be difficult but of paramount importance as withdrawal of the offending drug is the sole management for the former whereas immunosuppressive therapy, anti-inflammatory drugs and sometimes plasmapheresis may be needed in the later. Drugs of almost every class can lead to vasculitis; antibiotics (cephalosporins, penicillin, nitrocycline, fluoroquinolones, gentamicin, tetracycline, vancomycin), antivirals, antithyroid (carbimazole, propylthiouracil), anti-TNF $\alpha$ agents (infliximab, etanercept), psychoactive agents (clozapine,thioridazine), phenytoin, allopurinol, anticoagulants, analgesics, cardiovascular agents, antidiabetics, antineoplastics, etc.(Radic et al 2012). Due to the absence of reliable confirmatory tests DIV is often considered a diagnosis of exclusion.

Branka Bonaci et al highlighted the following differences between drug induced vasculitis (DIV) and idiopathic vasculitis (IV): 
a) Cutaneous vasculitis is more common in patients with DIV $(63 \%)$. Only $25 \%$ of patients with IV have cutaneous involvement.

b) Renal vasculitis is more common in IV (75\%). Only $19 \%$ of patients with DIV have renal involvement.

c) Patients with DIV are mostly positive for myeloperoxidase ANCA, antinuclear antibody (ANAs), antihistone and anticardiolipin antibodies and high levels of IgM and low C4 levels may be present. Patients with IV test negative for ANAs, antihistone and anticardiolipin antibodies and have normal $\mathrm{C} 4$ levels.

The contrasting feature in our patient was that he tested negative for ANCA which is not a characteristic of DIV as described by previous studies. (Bonaci et al 2005, Herlin et al 2002). Most of the studies on DIV have been conducted on antithyroid drugs and this may be one of the reasons for the discrepancy (Herlin et al 2002). It is not uncommon to have DIV with negative ANA and ANCA levels as has been reported with the use of ciprofloxacin (Storsley et al 2007).

Embolism, thrombosis, vasculitis, atherosclerosis, arteriosclerosis, raynauds phenomena, traumatic injuries and circulatory abnormalities can compromise vascular supply of a limb. None of them were present in our patient. Dry gangrene of limbs is characteristic of medium vessel vasculitis like Buerger's disease and polyarteritis nodosa which were also ruled out in our patient. Diagnosis of DIV was reached after excluding all the above mentioned etiologies and on the basis of temporal relation of use of drug and development of gangrene. Further extension of gangrene was limited after withdrawal of the drug and this again supports our diagnosis. Drug induced vasculitis and idiopathic SLE are difficult to differentiate clinically. Anti dsDNA antibodies and immune complexes are present in SLE. Small vessel vasculitis

(Hypersensitivity vasculitis or cutaneous leukocytoclastic vasculitis) is caused by drug hypersensitivity reactions and numerous other pathologies namely collagen vascular disorders (rheumatoid arthritis, Sjögren's syndrome, SLE), inflammatory bowel diseases, infections, henoch schonlein purpura, etc. Diagnosis of hypersensitivity vasculitis is made on the basis of criteria defined by American college of Rheumatology (Calabrese et al 1990). (Table $1)$.

Haber et al has reported DIV in patients ranging from 2 days to 9 years after exposure to offending drug.

Table 1: American College of Rheumatology Criteria for Hypersensitivity Vasculitis

\begin{tabular}{ll}
\hline 1 & Age $>16$ years at disease onset \\
\hline 2 & Medication at disease onset as precipitating factor \\
3 & Palpable purpura \\
4 & Maculopapular rash \\
5 & Skin biopsy with granulocytes around an arteriole or venule \\
Diagnosis is made when $>3$ criteria are present (sensitivity $71 \%$; specific- \\
ity $84 \%)$
\end{tabular}

DIV may have dermatologic involvement in the form of palpable purpura, maculopapular rash, haemorrhagic blisters, pustules or erosions. The lesions characteristically affect the lower limb and may be associated with systemic features like fever, malaise, arthralgia, etc. Involvement of kidneys leads to glomerular vasculitis or end stage kidney disease whereas involvement of lungs leads to haemorrhagic syndrome. DIV secondary to levamisole is characterised by purpura affecting external ears, nose and cheeks whereas vasculitis secondary to gemcitabine is characterised by extensive necrosis in muscle and gastrointestinal tract. (Tsai et al 2013, Eyre et al 2014)

Management of DIV is withdrawal of the offending agent. Anticoagulant and anti-inflammatory properties of low-molecular weight heparin limit further extention of vascular thrombosis in patients with critical ischaemia.(Harada et al 2011). Immunosuppressive therapy is administered in cases with vital organ involvement. Use of synthetic analogues of prostacyclin like epoprostenol (Iloprost) is advocated in patients with critical peripheral ischemia (CPI) secondary to autoimmune rheumatic disease
(ARD). Therapeutic advantage can be explained on the basis of vasodilatory, antithrombotic, fibrinolytic and anti-inflammatory action. (Jeffery et al 2008). B-cell depletion therapy with rituximab has also been proposed in patients with severe ischaemia not responding to epoprostenol. (Rubenstein et al 2006).

Quinolones or third-generation cephalosporins are first line agents for management of typhoid fever. But combination of chloramphenicol and gentamicin or cefotaxime and amikacin has been used successfully in patients resistant to quinolones (Das 2000). Bone marrow suppression (reversible with cassation of therapy), chloramphenicol induced aplastic anaemia (CIAA), Grey baby syndrome are some of the unique adverse effects reported with the use of this drug (Balbi 2004).

\section{Conclusion}

High index of suspicion is needed to detect a DIV. Withdrawal of the offending agent is the only definitive management. Monitoring of ANCAs, ANAs and anticardiolipin antibodies is advisable in serologically positive patients. This is the first case report of ANCA negative DIV secondary to oral intake of chloramphenicol

\section{References}

[1] Edwards IR and Aronson JK (2000), adverse drug reactions: definitions, diagnosis, and management, Lancet. 356(9237)1255-9. http://dx.doi.org/10.1016/S0140-6736 (00)02799-9.

[2] Jennette JC, Falk RJ, Andrassy K, Bacon PA, Churg J, Gross WL, et al. (1994), Nomenclature of systemic vasculitides. Proposal of an international consensus conference. Arthritis Rheum.37:18792.http://dx.doi.org/10.1002/art.1780370206.

[3] Doyle MK and Cuellar ML (2003), Drug-induced vasculitis. Expert Opinion on Drug Safety. 2(4) 401-409. http://dx.doi.org/10.1517/14740338.2.4.401.

[4] Radić M, Martinović Kaliterna D, Radić J (2012), Drug-induced vasculitis: a clinical and pathological review. Neth J Med. 70 (1) 12-7.

[5] Bonaci-Nikolic BM, Nikolic MM, Andrejevic S, Zoric S, Bukilica M. (2005), Antineutrophil cytoplasmic antibodies (ANCA)-associated autoimmune diseases induced by antithyroid drugs: Comparison with idiopathic vasculitides. Arthritis Res Ther, 7 (5) R1072-R1081 http://dx.doi.org/10.1186/ar1789.

[6] Herlin T, Birkebaek NH, Wolthers OD, Heegaard NH, Wiik A.(2002) Antineutrophil cytoplasmic antibodies (ANCA) prophiles in propylthiouracil-induced lupus-like manifestations in monozygotic triplets with hyperthyroidism. Scand J Rheumatol, 31 (1) 4649.http://dx.doi.org/10.1080/030097402317255381.

[7] Storsley L and Geldenhuys L.(2007) Ciprofloxacin-induced ANCAnegative cutaneous and renal vasculitis--resolution with drug withdrawal.Nephrol Dial Transplant.,22(2) 660-1. http://dx.doi.org/10.1093/ndt/gf1554.

[8] Calabrese LH, Michel BA, Bloch DA, Arend WP, Edworthy SM, Fauci AS,et al. (1990) The American College of Rheumatology 1990 criteria for the classification of hypersensitivity vasculitis. Arthritis Rheum 1990 ; 33(8)1108-1113 http://dx.doi.org/10.1002/art.1780330808.

[9] 9. Haber MM, Marboe CC, Fenoglio JJ. Vasculitis in drug reactions and serum sickness. In: Churg A, Churg J (eds). Systemic Vasculitides. New York: Igaku-Shoin; 1991. pp. 305-311.

[10]Tsai MH, Yang JH, Kung SL, Hsiao YP. (2013) Levamisole-induced myopathy and leukocytoclastic vasculitis: a case report and literature review. Dermatol Ther. $26 \quad$ (6) 476-80 http://dx.doi.org/10.1111/dth.12018.

[11]Eyre TA, Gooding S, Patel I, Moore N, Hatton C, Collins GP. (2014) Gemcitabine-induced large vessel vasculitis demonstrated by PET CT: a rare, important side effect. Int $\mathrm{J}$ Hematol. 99(6):798-800 http://dx.doi.org/10.1007/s12185-014-1555-5

[12]Harada N, Okajima K, Uchiba M. (2006) Dalteparin, a low molecular weight heparin, attenuates inflammatory responses and reduces ischaemia-reperfusion-induced liver injury in rats. Crit Care Med 2006; 34(7) 2011-3. http://dx.doi.org/10.1097/01.CCM.0000220764.10155.03.

[13]Jeffery RC, Narshi CB, Isenberg DA. (2008) Prevalence, serological features, response to treatment and outcome of critical peripheral ischaemia in a cohort of lupus patients. Rheumatology, 47 (9) 1379-83. http://dx.doi.org/10.1093/rheumatology/ken210. 
[14]Rubenstein E, Arkfeld DG, Metyas S, Shinada S, Ehresmann S, Liebmann H. (2006), Rituximab treatment for resistant antiphospholipid syndrome. J Rheumatol, 33(2) 255-7.

[15]Das U, (2000), Multidrug resistant Salmonella typhi in Rourkela, Orissa. Indian J Pathol Microbiol, 43(2)135-8.

[16]Balbi H (2004) Chloramphenicol: A Review. Pediatrics in Review, 25:284-88. http://dx.doi.org/10.1542/pir.25-8-284. 